\title{
ANALYSIS OF GAMIFICATION ELEMENTS TO EXPLORE MISINFORMATION SHARING BASED ON U\&G THEORY: A SOFTWARE ENGINEERING Perspective
}

\author{
Malik Malki and Amin Shaqrah \\ College of Computer Science \& Engineering in Yanbu- Taibah University, \\ Medina, Saudi Arabia
}

\begin{abstract}
Gamification elements provide apersonal drive to urge user experience, emotion, fun, and engagement, positively or negatively. These gamification elements may have been unintentionally employed through the designand implementation processof social media platforms to encourage users' behaviour towards misinformation sharing. This study intends to answer the subsequent question” What are the mostly used gamification elements that could possibly encourage users to share misinformation on social media platforms?". The study empirically investigates the usage of gamification elements and their relation to $U \& G$ theory with 286 participants. The results indicated that gamification elements usage scored high with regard tothe self-expression perspective (frequency=216), as well as the interaction \& collaborations perspective (frequency=198). Where as, the information seeking perspective scored low (frequency=59) and leaderboard were the least usage(frequency=43). The results may be useful to guide software engineering, developers, GUI specialists to cater for design elements settings and their possible negative effects in social media contexts.
\end{abstract}

\section{KEYWORDS}

Online misinformation, Gamification elements, Software engineering, UGT.

\section{INTRODUCTION}

Social media platforms- such as WhatsApp, Facebook, Twitter, etc- are online software applications that simplify communicate, create, and disseminate user-generated content and becoming widely used as an important source of information [1]. These social media platforms utilized by users to share and spread information quickly and timely for many reasons. Unfortunately, social media platforms misuse was growing widely and rapidly [2]. However, a serious challenge is that the information generated and communicated through theses social media platforms is not always credible [3], [4]. [5] concluded that Facebook usage is a user's motivation and expressively associated with time spent on Facebook as a salient. [6] discussed the spread of online misinformation as one of the recent trends that we need to be aware of. A recent study on misinformation on social media platforms [1]showed that about (67\%) of users specified that they had shared misinformation on social media themselves, (94\%) indicated that they had seen other users share misinformation on social media.

Misinformation can appear on social media in numerous types like "rumours, buzzes, factoids, etc". Nevertheless, the results are imprecise information that is spread purposely or inadvertently [2]. Spreading misinformation confuses the users and can promote illegal usage that are harmful to individuals [7]. Additionally, spreading misinformation can highly hinder the credibility of 
social media's information content. This gives a rich view point of the seriousness of misinformation spreading on social media platforms and not only advocates the need to act to minimize its negative effect on users, but also to safeguard the quality of the generated and communicated information through these platforms. In the recent years, several researches from computer science and information system field studied misinformation aiming to develop algorithms to detect low quality information[4]. Another related research observed the impact of user-intrinsic influences (personality and internal drive) and its possible effect on users spreading misinformation on social media [8]. However, none of these studies focus on how to minimise the act of misinforming spreading or sharing among social media users. Luckily, software as a medium for misinformation spread may be a channel for enacting a behaviour change strategy to minimise the act of misinformation spreading on social media platforms. This can ultimately minimize the negative effect of misinformation spreading on users and enhance information value and on different social media platforms.

Gamification refers to the adoption of game design elements (e.g. badges, points, levels, leaderboard) in non-game contexts and has been used as an active behaviour change tactic to motivate users for the purpose of changing their behaviours towards desired ones [9], [10]. The common use of gamification is to take the scoring elements of games such as - points, badges, levels, leaderboards- and deploy them to a non-game context such as: educational or business context[11].Numerous applications of gamification inspire various goals in several environments, such as adopting a better and healthy lifestyle, enhancing students' awareness with class activities to attain better grades, or enhancing quality and efficiency of business environment [12], [13], [14], [15]. For instance, points and leader-boards can used in call centres to increase employees' satisfaction- the number of calls taken, the number of problems solved, the time taken for finishing a task, and the customers' overall satisfaction- [15].

This improves the process of generating and sharing information on social media by motivating users to share and generate high quality content in a gamified and more enjoyable environment. For example, a user can gain or lose points on the quality of information individuals shares based on the users'ratings. Nevertheless, the design of gamification can playa major effect on the individuals performance compared to a non-gamified context[16]. This highlights the need to study and investigate how gamification can systematically be adopted in the context of misinformation on social media platforms. In this study, the authors quantitatively investigate the usage of gamification elements and their relation to $U \& G$ theory in the design of social media platform and their possible effect on users' behaviour to share misinformation. The outcome of this study is meant to help software engineers to design gamified social media applications that are misinformation-aware. This will help improving users' behaviour towards misinformation sharing on social media platforms.

This study is structured as follows. In next section the authors discussed the literature review. In section 3the used methodology presented and discussed. Then, a proposed gamification elements matrix was developed in section 4.Discussion and conclusion are in the last section.

\section{LITERATURE REVIEW}

\subsection{Gamification Elements}

The most common elements in gamified social software are: (1) points(PO): the main reward users can acquire from their actions will be in the form of points, the more substantial result accomplished by a user in an action, the higher the number of collected points as a reward. (2) levels (LE): The level function mapping the amount of points to levels incrementally; achieving the next level becomes harder as a progress, the amount of actions and next stage to finishing a 
specific goal is stated [17]. (3) badges (BA): will be used as a reward to distinguish of certain stages achievement a progress.[18] noted various badges especially the online badges being granted do not need abundant time or effort. (4) A leaderboard (LB) means which users can track their achievements comparing to others and presentation a user stands regarding to others. Leaderboards can be demised into several smaller groups[19]. (5) widget (WI): is a feature added to a social software game, these give additional features and capabilities that provides an overall better experience, widget illustrated the top-scoring users and the rank. (6) reward \& punishment(RP): is an achievement evaluation -positive or negative-which is obtained by the users because of the appraisal of mission affording to the game rules. Achievement require users to access the system or flow or take some action at a time or place for either a positive effect or to avoid a negative effect. (7) interaction \& collaborations (IC): Interaction \& collaborations will be reinforced in the gamified social media software, according to [19]" the player site will also act as a social network, in which players can have friends and can chat or send messages to them". Furthermore, this social network also affects other game procedure such as rankings, so players will see some ranking permit players to compare their results with those attained by others[20].

\subsection{U\& G Theory}

Uses and Gratifications (U\&G) theory well-recognized on users' motivations and adopted by researchers [21], [22], [23]. Four motivation types in social media and well-known by the U\&G theory[21], [22]. (1) entertainment, this type is concern with utilize social media for special and personal entertain; [23] detected the incremental increase in users require entertaining on social media. [24] detected that sharing photo on Flickr, users liked this action apart from significances. (2) social motivations, Social motivation candidate as the main reason causing the acceptance of social media [25]. [21] found the socializing once promoting actions with a few clicks is a relaxed way that permitted users to connect and chatting with others, likewise motivation for sharing news on social media (3) information seeking, information seeking emphases on matching informational desires with social media applications; information seeking became an important motivation of sharing news on social media [21]. Whereas misinformation is inexact, misinformation likely that users share it probably to find more explanation from social networks. (4) self-expression means utilizing social media to prompt oneself and get the reputation,users sharing news on social media to empower others enter the content and to accomplish status in place [21]. [22] and [23] described U\&G theory as one of the most recognized approach to comprehend users' motivation of social media usage and has been generally accepted.

[26]summarized the gamification elements can be problematic at design and implementing stage even some researchers might think that game elements are easy. [27] defined motivation and divided into two different types: extrinsic and intrinsic. Extrinsic motivation is defined as behavior determined by external reward or punishment; whereas intrinsic motivation is defined as behavior determined by personal drive or gratification. A key reflection for gamification developers should be embrace game elements that stimulate individuals extrinsically and intrinsically. While several concerns which individuals using gamification require consideration, there are several clarifications to confirm gamification's usefulness. [28] found many social media applications are currently using rewards and competitive tactics generally found in the gaming world to make users' actions more entertaining, while it is not entirely new idea that applying game elements can encourage misinformation sharing. This implies that game dynamics act as motivational sources and are not limited to extrinsic rewards.

Software engineering designers seeking ways to boost user engagement can take advantage of this awareness by enabling users to share accurate information. Users disseminate misinformation when they forward misinformation to their own social networks innocently. Consequently, users could credibly take ladders to realize the information to be inaccurate. Social media may increase the spread of misinformation for many reasons. First, lack of solid quality 
controlling on social media mechanisms contrasting traditional media. Second, social media applications make it relaxed to broadcast information, as well as misinformation, a mouse click user can forward messages to many receivers quite smoothly. Third, misinformation on social media can rapidly spread many users, which can cause misunderstanding and redundant apprehension among public domain[7].

\section{METHOD}

Students at Taibah University (Faculty of computer sciences and engineering in Yanbu) have been selected as the main sampling participants in 2018/2019 first semester. The participants are pioneered of gamifications use, not only for the learning purposes but also it has become part of their lifestyle. Additionally, they are heavy users of the latest mobile devices. Hence, they are anappropriate target for this study.A sample of students was selected from WhatsApp groups during 2018-2019 period, about 450 undergraduate students were invited to finishing a survey regarding gamification elements and misinformation sharing.

An online questionnaire was suitable for the present study as it is appropriate for gathering students' attitudes and behavior. Misinformation was defined in this study as imprecise information. During data gathering(370)students'responses were collected with a response rate of 82.2\%.290 of which finished the survey and (3) were unfinished with (1) answer missing separately. Finally, gathered responses (286) were used for our analysis. All the faculty students selected from faculty of computer sciences and engineering in Yanbu, so (150) out of (286) were ranging from (19) to (24) years old, (120) were under (19) years old- preparatory year- and (16) were above (25) years old -Tajseer students-. Since the data were collected from Saudi Arabia, the selected students were mainly from Medina county community.

\section{RESULTS}

This study aims to prioritize the importance of gamification elements that should be considered by gamification designers based on users' behaviors, starting with literature to capture gamifications elements and $U \& G$ theory. The authors devised a matrix that can serve as a guide to software designers during the design of gamification elements and provide explanation and assessment of their usefulness. Gamification elements and mechanics had been developed to increase users' motivation to share information, participation and learning. Positive elements of gamification yield benefit to users' motivation to do the best and vice versa. [29]investigated gamification in social interaction and determined that gamification is grounded in artificial game mechanics -points, dashboard, leaderboard, levels, badges, punishment, content unlocking, currency-and that more elements should be studied in software projects. [19]concluded that the implementation of gamification in software engineering is moving slower than in other fields i.e. education, community management, health, banking, librarians, networking, and marketing.

The main study question focus on which gamification elements may encourage people to share misinformation based on the four $U \& G$ motivations. The matrix (see figure1) illustrates the common gamification elements usage (1.Points, 2. Levels, 3.Badges, 4.Leaderboards, 5. Widgets,6. Rewards \& punishment, 7. Interaction \& collaborations) in relation to the U\&G perspectives. In general, students voting results of gamification elements on the four U\&G perspectives seems fit as seen in figure 1 . 


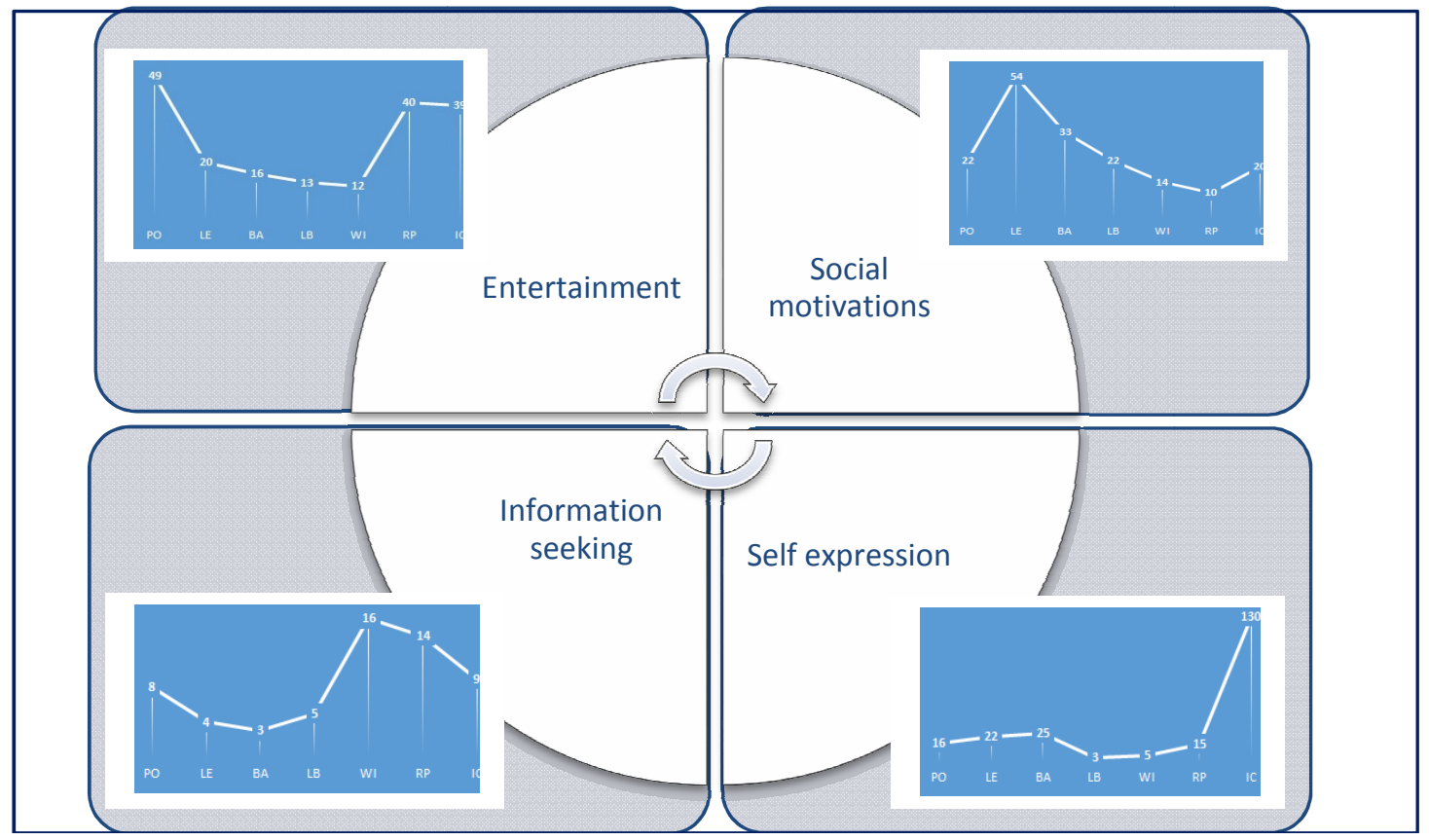

Figure 1.The matrix of gamification elements based on UGT*

*Po=points, $\mathrm{LE}=$ levels, $\mathrm{BA}=$ badges, $\mathrm{LB}=$ leaderboard, $\mathrm{WI}=$ widget, $\mathrm{RP}=$ reward \& punishment, $\mathrm{IC}=$ interaction\& collaborations

Table 1. shows that the number of responses on interaction \& collaborations element was (198). It also shows that the most applied gamification elements for misinformation sharing is in selfexpression perspective as the number of responses was (216). The second, third, and fourth most used gamification element were levels, points, and reward \& punishment systems. Levels and points increase students' participation in misinformation. Other gamification elements such as badges, leaderboard, and widget are less used.

\section{Discussion AND Conclusion}

Due to the notable spread of misinformation these days, it is imperative to identify the motivations behind the act of misinformation sharing on social media. Some of this misinformation sharing might be avoided by software engineering and game designers at the designing and developing stage of social media platforms. The four motivations of U\&G theory

Table 1. Voting of the usage of gamification elements on misinformation sharing results*

\begin{tabular}{|l|l|l|l|l|l|l|l|l|}
\hline \multicolumn{1}{|c|}{ G. element } & Po & LE & BA & LB & WI & RP & IC & Total voting \\
Perspective & & & & & & & & \\
\hline Entertainment & 49 & 20 & 16 & 13 & 12 & 40 & 39 & 189 \\
\hline Social motivations & 22 & 54 & 33 & 22 & 14 & 10 & 20 & 175 \\
\hline Information seeking & 8 & 4 & 3 & 5 & 16 & 14 & 9 & 59 \\
\hline Self-expression & 16 & 22 & 25 & 3 & 5 & 15 & 130 & 216 \\
\hline Total voting & 95 & 100 & 77 & 43 & 32 & 79 & 198 & \\
\hline
\end{tabular}

*Po=points, $\mathrm{LE}=$ levels, $\mathrm{BA}=$ badges, $\mathrm{LB}=$ leaderboard, $\mathrm{WI}=$ widget, $\mathrm{RP}=$ reward $\&$ punishment, $\mathrm{IC}=$ interaction\& collaborations 
have been found substantial in wide-ranging fields. This study aims to examine their suitability with regard to the context of gamification for misinformation sharing on social media platforms.

Beyond examining users' motivations as illustrated by U\&G approach, this study examines the elements of gamification, which might encourage users' choices to share misinformation on social media too. That means the investigation of gamification elements that encourage users to share misinformation based on U\&G approach as follows: entertainment "which gamification elements affect users to share misinformation for entertaining their needs, users found fun and novel form that can be gamely enjoyed"; Social motivation" which gamification elements affect users to share misinformation for building and maintaining interpersonal relationships. Users found entertaining since the social media sharing mechanism is comfortable with just a few seconds, and it lets users to socialize with each other's e.g. share with comment that asks for friends' contribution; information seeking "which gamification elements affect users to share misinformation for informative needs"; and self-expression" which gamification elements affect users to share misinformation for gaining reputation among others".Users found sharing misinformation can lead to achieving self-status; for example, to get more "likes" and consideration from others by sharing entertaining or information originator [8]. Users tends to forward post using elements that they find entertaining, while others select another element to forward post. These elements were measured thought U\&G motivation of users' misinformation sharing theory.

This study adopted grounded theory which absorbed on inductive strategies for data analysis. It focuses on abstract perceptions and clarify data results. [30] summarized this method as a method that starts and ends with data. Software engineering practicallyuses of gamification elements for many different purposes and they should considerwhich elements had the most impact on users'attitudes and behaviors to share in accurate or false information. Seven gamification elements are the most used in Saudi Arabia environments and are ordered as follows:interaction $\&$ collaborations, levels, points, reward \& punishment, badges, leaderboard, and widgets. On the other side, the use of gamification elements has been pragmatic from the four UGT perspectives. In general, the self-expression perspective was commonly used for misinformation sharing, then entertainment and social motivations, and information seeking were the least perspective.

\section{REFERENCES}

[1] X. Chen, and S. Sin, “Misinformation? What of it?'Motivations and individual differences in misinformation sharing on social media," Proceedings of the Association for Information Science and Technology, Vol. 50(1), 2013.

[2] N. A. Karlova, and K. E. Fisher, "A social diffusion model of misinformation and disinformation for understanding human information behavior. Information Research," Vol. 18 (1), Retrieved from http://informationr.net/ir/18-1/paper573.html, 2013.

[3] O. Oh, M. Agrawal, and H. Rao, "Community intelligence and social media services: a rumor theoretic analysis of tweets during social crises,” MIS Quarterly, Vol.37(2), 2013.

[4] K. Starbird, J. Maddock, M. Orand, P. Achterman, and R. Mason, R, "Rumors, false flags, and digital vigilantes: Misinformation on Twitter after the 2013 Boston marathon bombing," iConference Proceedings, 2014.

[5] C. Ross, E. Orr, M. Sisic, M. Jaime, J. Michelle, and M. Simmering, "Personality and motivations associated with Facebook use," Computers in Human Behavior, Vol. 25 (2009),2019.

[6] World Economic Forum, "Top 10 trends of 2014. The rapid spread of misinformation online," Retrived from http://bit.ly/1edZQQF, 2014. 
International Journal of Software Engineering \& Applications (IJSEA), Vol.10, No.4, July 2019

[7] C. Budak, D. Agrawal, and A. Abbadi, "Limiting the spread of misinformation in social networks," Proceedings of the 20th International Conference on World Wide Web, pp. 665-674, 2011.

[8] X. Chen, "The influences of personality and motivation on the sharing of misinformation on social media," IConference Proceedings,2016.

[9] S. Deterding, D. Dixon, R. Khaled, and I. Nacke, "From game design elements to gamefulness: defining gamification," in Proceedings of the 15th International Academic MindTrek Conference: Envisioning Future Media Environments, 2011.

[10] P. Herzig, M. Ameling, and A. Schill, "A Generic Platform for Enterprise Gamification," In Proceedings of Software Architecture (WICSA) and European Conference on Software Architecture, (pp. 219-223), 2012.

[11] S. Nicholson, "A user-centered theoretical framework for meaningful gamification," Games Learning Society, Vol. 8(1), 2012.

[12] S. O’Donovan, J. Gain, and P. Marais,"A case study in the gamification of a university-level games development course," In Proceedings of the South African Institute for Computer Scientists and Information Technologists Conference, 2013.

[13] J. Simões, R. Redondo, and A. Vilas, "A social gamification framework for a K-6 learning platform," Computers in Human Behavior, Vol. 29(2), 2013.

[14] K. Robson, K. Plangger, J. Kietzmann, I. McCarthy, and L. Pitt, "Game on: Engaging customers and employees through gamification,” Business Horizons, Vol. 59(1), 2016.

[15] L. Rodrigues, A. Oliveira, and C. Costa, "Playing seriously - How gamification and social cues influence bank customers to use gamified e-business applications," Computers in Human Behavior, Vol. 63, 2016.

[16] F. Dalpiaz, R. Snijders, S. Brinkkemper, M. Hosseini, A. Shahri, and R. Ali, "Engaging the crowd of stakeholders in requirements engineering via gamification," Springer International Publishing, 2017.

[17] P. Sweetser, and P. Wyeth, P. "Game Flow: A model for evaluating player enjoyment in games," Computation Entertain-Theoretical Practical Computation Applications Entertain, Vol.3(3), 2005.

[18] A. Halavais, "A genealogy of badges: Inherited meaning and monstrous moral hybrids," Information, Communication and Society, Vol.15(3), 2012.

[19] F. Garcia, O. Pedreira, M. Piattini, and M. Penabad, "A framework for gamification in software engineering," The Journal of Systems and Software, Vol. (132), 2017.

[20] Y. Yang, A. Yousra, and D. Yogesh, "Examining the impact of gamification on intention of engagement and brand attitude in the marketing context," Computers in Human Behavior, Vol. (73), 2017.

[21] C. Lee, and L. Ma, "News sharing in social media: The effect of gratifications and prior experience," Computers in Human Behavior, Vol. 28(2), 2012.

[22] N. Park, K. Kee, and S. Valenzuela, "Being immersed in social networking environment: Facebook groups, uses and gratifications, and social outcomes," Cyber Psychology \& Behavior, Vol. 12(6), 2009.

[23] G. Shao, G. "Understanding the appeal of user-generated media: A uses and gratification perspective," Internet Research, Vol. 19(1), 2009. 
International Journal of Software Engineering \& Applications (IJSEA), Vol.10, No.4, July 2019

[24] O. Nov, M. Naaman, and C. Ye, "Analysis of participation in an online photo-sharing community: A multidimensional perspective," Journal of the American Society for Information Science and Technology, Vol. 61(3), 2009.

[25] J. Kim, M. Kim, and Y. Nam, “An analysis of self-construal, motivations, Facebook use, and user satisfaction,” International Journal of Human Computer Interaction, Vol. 26(11-12), 2010.

[26] A. Dorling, and F. McCaffery, "The gamification of SPICE," International Conference on Software Process Improvement and Capability Determination, Springer, 2012.

[27] T. J. Brigham, “An Introduction to Gamification: Adding Game Elements for Engagement,"Medical Reference Services Quarterly, Vol. 34(4), 2015.

[28] R. Silverman, R. "Latest game theory: mixing work and play,” Wall Street Journal, 2011.

[29] Y. Xu, "Literature review on web application gamification and analytics," Honolulu, HI, pp.11-05, 2011.

[30] K. Charmaz, "Constructing grounded theory: A practical guide through qualitative analysis," 2nd ed. London: Sage, 2006.

\section{AUTHORS}

Malik Malkiis an Assistant Professor of Software Engineering at the College of Science and Computer Engineering, Taibah University, Yanbu. He received his BSc in Computer Science from Taif University, KSA, in 2008 and his MSc in Advanced Software Engineering from Leicester University, UK, in 2011. He also received a PhD in Software Engineering from Bournemouth University, UK, in 2015. His research is focused on the engineering of social informatics, i.e., the systematic design of softwarebased solutions taking into consideration their interactions with related institutional and cultural contexts.

Amin Shaqrah is currently Associate Professor of Information Systems at Taibah UniversitylSaudi Arabia. Shaqrah received his $\mathrm{PhD}$ in MIS from University of Banking \& Financial Sciences and received MA in MIS from Amman Arab University for Graduate Studies. He had a leadership role in the design and implementation of MIS program at the undergraduate/graduate level. He is affiliated with several International professional societies on knowledge management, E-business, and a member of editorial review boards for a number of International Journals. His research interests include knowledge sharing and transfer, CRM value strategies, IT/IS adoption, human and social implications of enterprise systems (KM, CRM, and BI).

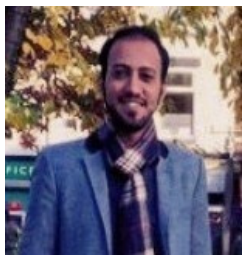
His research work has appeared in several leading International Journals and conferences.

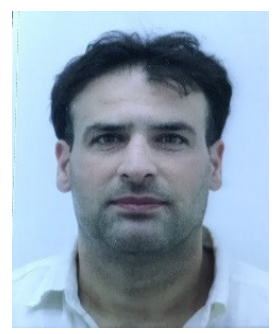

